\title{
Multiwavelength Operation of Erbium-Doped Fiber Lasers by Periodic Filtering and Phase Modulation
}

\author{
Mohammad Aleem Mirza and George Stewart
}

\begin{abstract}
This paper explains the principles behind multiwavelength operation of an erbium-doped fiber laser (EDFL) under the combined effect of cavity phase modulation and periodic wavelength filtering. A mathematical model is developed to simulate the behaviour of the multiwavelength EDFL, both in time and frequency domains. The effects of various parameters such as pump power, filter channel spacing, modulation index and frequency are observed and explained. An all-fiber EDFL was constructed, using a piezo-transducer-based phase modulator and a Sagnac loop periodic filter, to validate the theoretical results. The effects of pump power, modulation frequency and modulation index were monitored experimentally, justifying the theoretical explanation. The multiwavelength EDFL has several potential applications in fiber sensing due to its flexible all-fiber design.
\end{abstract}

Index Terms-Erbium-doped fiber lasers (EDFL), multiwavelength fiber laser, phase modulation, sagnac loop filter.

\section{INTRODUCTION}

$\mathbf{T}$ HE DEVELOPMENT of multiwavelength erbium-doped fiber lasers has attracted much research interest because of their potential use in optical communication, fiber sensors and spectroscopy. At room temperature an EDFL cavity can only oscillate at one wavelength at any particular time because the gain spectrum of the erbium-doped fiber amplifier (EDFA) is predominantly homogeneously broadened. One method to achieve multiwavelength operation is cooling the EDFA to $77^{\circ} \mathrm{K}$ in liquid nitrogen to reduce the homogeneous line-width and using wavelength selective filters [1]-[3] but this technique is inconvenient. Alternatively a twin-core erbium-doped fiber (EDF) may be used [4] to provide an inhomogeneous gain medium at room temperature; however the EDF design is very complex. Several other methods have been proposed to obtain multiwavelength operation at room temperature by adding Raman amplification [5], stimulated Brillouin scattering (SBS) [6], [7] or a semiconductor optical amplifier (SOA) [8] in an EDFL cavity. Such designs, nevertheless, are not purely EDFL systems because multiple wavelengths are generated and amplified by the hybrid gain medium. A multiwavelength laser, designed for room temperature operation with only EDF as a gain medium, needs two basic elements: 1) a wavelength selective filter and 2) an "active element" to continuously share an almost uniform gain among the selected wavelength channels, thus counteracting the cross gain saturation in the EDFA. A

Manuscript received November 16, 2007; revised May 15, 2008. Current version published April 17, 2009.

The authors are with the Centre for Microsystems and Photonics, Department of Electronic and Electrical Engineering, University of Strathclyde, Glasgow G1 1XW, U.K. (e-mail: aleem.mirza@eee.strath.ac.uk; g.stewart@eee.strath.ac.uk).

Digital Object Identifier 10.1109/JLT.2008.2009160 highly nonlinear photonic crystal fiber (PCF) has been used [9], [10] as an active element, along with a periodic filter, to produce a multiwavelength EDFL but the design needs a high power EDFA to initiate the four-wave-mixing (FWM) in the PCF. A simpler method for a multiwavelength EDFL is intra-cavity frequency or wavelength shifting, which can be achieved through an acousto-optic frequency shifter [11] or a phase modulator [12]-[15].

The use of a simple piezo-transducer (PZT) phase modulator along with periodic filtering is a particularly simple and low-cost design for a room-temperature multiwavelength laser with a number of potential applications. However, while previous work has experimentally demonstrated this type of system and observed that the multiwavelength operation is critically dependent on the choice of certain modulation parameters [12]-[14], a full theoretical understanding of its operation based on fiber laser dynamics has not been presented. Previous work has demonstrated only experimental results with a brief explanation but the actual dependence of multiwavelength operation on all the design parameters of the EDFL has not been, to date, determined quantitatively. For example, it is stated in [12] that a multiwavelength spectrum is achieved when the phase modulation frequency is comparable to the relaxation oscillation frequency, whereas [14] emphasizes the use of a sine or saw-tooth waveform and controlling the modulation index such that the dc component of the modulated field is minimized. On the contrary, square wave modulation is preferred in [13]. The effects of cavity loss, pump power and the filter's channel spacing have not been addressed at all. Moreover, only the spectral domain output has been previously discussed with no consideration of the corresponding time domain output, both during multiwavelength operation and when multiwavelength operation is inhibited.

This paper seeks to address these issues and identify the conditions under which multiwavelength operation is expected. A mathematical model is developed to analyze the effects of all the parameters, including the parameters of the phase modulator, the periodic filter and the laser cavity on multiwavelength operation of the EDFL. The model is based on the atomicand cavity-rate equations [18], specifically developed for fiber lasers with large cavities (where the longitudinal mode spacing is small, typically several MHz), including the effects of spontaneous and amplified spontaneous emission (ASE). The coupled rate equations are numerically solved to produce a time-invariant steady-state output power under the combined effect of sinusoidal phase modulation and periodic filtering from a Sagnac loop filter (SLF). The simulations of the mathematical model have shown that multiwavelength operation cannot be simply controlled by choosing a particular modulation index 


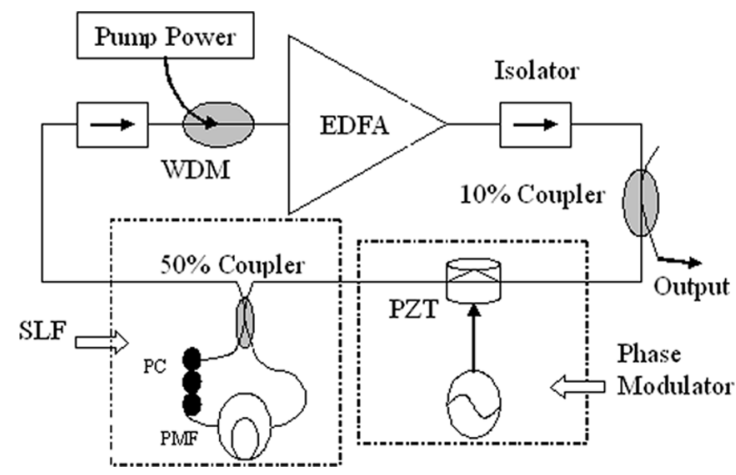

Fig. 1. Schematic of the multiwavelength EDFL design.

or frequency as claimed by the authors of the similar design [12]-[14], rather it strongly depends upon the combination of pump power, modulation frequency and index, filter response and the net closed-loop gain of selected modes. An analytical solution is also derived under the valid approximation of slow modulation rate as compared to the round-trip time of the ring cavity. Thus, for selected multiwavelength channels the increase in optical power has been expressed through one differential equation. Such an equation, quantitatively expressing the effect of all the parameters on the gain of every axial mode during the multiwavelength operation, has not been reported before and is extremely valuable in understanding the operation of, and in designing a multiwavelength EDFL. The simulation results show that the output of the multiwavelength EDFL is not continuous-wave $(\mathrm{CW})$ but takes the shape of a pulse, repeating at the modulation frequency when the pump power, cavity loss, channel spacing, modulation frequency and index are controlled carefully. If these parameters are not adjusted properly the time-domain response varies, while the multiwavelength spectrum may still exist. Although not discussed in previous papers [12]-[14], control of the temporal behaviour of the multiwavelength EDFL, is very important before considering its use for any particular application. An experimental EDFL system was then constructed, using a SLF of fixed channel spacing $(0.8 \mathrm{~nm})$ and a PZT-based phase modulator, and the effects of pump power, modulation frequency and index were experimentally measured. The experimental results confirmed the theoretical model and demonstrated that multiple channels which share reasonably uniform gain from the EDFA produce stable, simultaneous and periodic pulses only when the pump power, modulation frequency and modulation index are properly set and controlled. The multiwavelength EDFL reported here has a flexible, all-fiber design, which can be adjusted to meet specific requirements in different types of applications.

\section{PRINCIPLES OF OPERATION}

\section{A. Role of Periodic Filter and Rate Equations (Without Phase Modulation)}

Fig. 1 shows the basic ring-cavity configuration of the EDFL system used in our studies. The EDFA is pumped by a $980 \mathrm{~nm}$ laser diode and can be conveniently described by a 2-level model [16], with the total gain from the EDFA proportional to the length-averaged inversion level, $\overline{N_{2}}(t)=(1 / l) \int_{0}^{l} N_{2}(z, t) d z$.

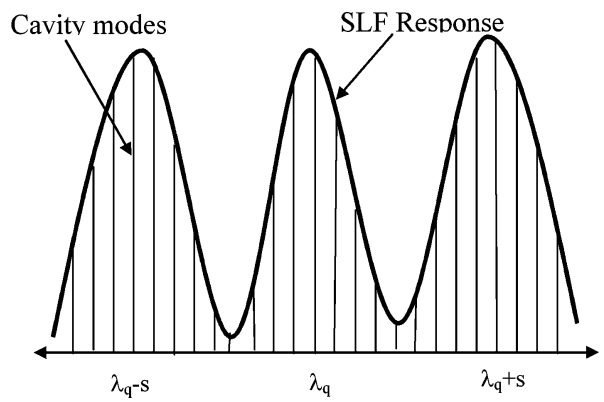

Fig. 2. Effect of an intra-cavity periodic filter on cavity modes.

The use of length-averaged gain does not affect our analysis because it is the net (or closed-loop) gain of selected cavity modes that determines the multiwavelength operation. The total gain provided by the EDFA to possible cavity modes can therefore be expressed as $\overline{g_{q}} \cdot l$, where $l$ is the length of the EDF and $\overline{g_{q}}=\left(\gamma_{q}+\alpha_{q}\right) \overline{N_{2}}-\alpha_{q}$ is the length-averaged gain coefficient, $\gamma_{q}$ and $\alpha_{q}$ are the emission and absorption coefficients for mode $q$.

In the absence of a periodic filter, the cavity loss $\alpha_{c}$ is fixed and the cavity mode with maximum gain would start lasing due to the homogeneous broadening of the EDFA. Due to the long length of the EDFL cavity (about $40 \mathrm{~m}$ ), the cavity modes are closely spaced (about $.04 \mathrm{pm}$ or $5 \mathrm{MHz}$ ) and a strong mode competition exists. However when a periodic filter is present in the cavity, the cavity loss becomes a function of wavelength and the net gain for the adjacent modes reduces. A Sagnac loop filter (SLF) is used as a periodic comb filter in Fig. 1, whose transfer function (ratio of output to input intensity) can be expressed as a function of wavelength [17]

$$
G_{f}(\lambda)=K_{f}\left[1-\cos \left(\frac{2 \pi}{\lambda} \cdot B \cdot L_{f}\right)\right]
$$

where $L_{f}$ and $B$ are the length and birefringence of the polarization maintaining fiber (PMF) used in the filter, and $K_{f}$ is the filter's scale factor that depends upon the orientation of the birefringent axes of the PMF with reference to plane of polarization. $K_{f}$ can be controlled by a polarization controller (PC) inside the SLF and its optimum value is 0.5 when the birefringent axes are at $45^{\circ}$ to the reference plane of polarization. The SLF introduces a wavelength dependent loss in the EDFL and it can be easily shown that the wavelength spacing, $s$, between adjacent channels (wavelengths at which $G_{f}$ is maximum) is $s=\lambda_{o}^{2} /\left(B \cdot L_{f}\right)$ where $\lambda_{o}$ is the centre wavelength of any channel and $G_{f}\left(\lambda_{o}\right)=1$ for the optimum setting of the PC. The effect of periodic filtering is conceptually drawn in Fig. 2, showing that the competition among adjacent cavity modes is suppressed and now the modes near the transmission peaks of selected channels will start competing, provided they get a uniform gain from the EDFA. Hence, the condition of uniform gain at the selected channels becomes a key criterion for a multiwavelength EDFL; however practically the EDFA gain cannot be ideally uniform and the laser will oscillate at the most favourable mode of all the filter-selected modes when no other measures are taken. 
Before we analyse the dynamics of the EDFL under the effect of intra-cavity phase modulation, the steady-state power of all the cavity modes have to be calculated by solving the coupled atomic and cavity rate equations. Equation (2) to (6), as given below, were derived in our recently published work [18] to compute the transient build-up of closely spaced axial modes when a step input is applied to the pump power. These equations are reproduced here, but now including the effect of periodic filtering from the SLF. The atomic rate equation for the EDFA is

$$
\begin{aligned}
(\rho S l) \cdot \frac{d \overline{N_{2}}}{d t}=P_{p} \cdot\left(1-e^{\overline{g_{p}} l}\right)-(\rho S l) \cdot \frac{\overline{N_{2}}}{\tau_{0}} \\
-\sum_{g} \frac{M_{g}}{\tau}\left(1-e^{-\overline{g_{g}} l}\right)-R_{\mathrm{ASE}}
\end{aligned}
$$

where $\rho$ is the erbium ion density, $\mathrm{S}$ is the cross-sectional area of the EDF core, $l$ is the EDF length, $\tau$ is the round trip time for the laser cavity, $\overline{N_{2}}, \overline{N_{1}}$ are length-averaged, fractional populations of the upper and ground states, $\overline{N_{1}}+\overline{N_{2}}=1$, and $\rho S l \overline{N_{2}}$ is the total number of erbium ions in the upper state and $\overline{g_{p}}=-\alpha_{p}\left(1-\overline{N_{2}}\right)$ is the length-averaged pump absorption coefficient. The second term on the right-hand side of (2) represents the spontaneous emission (SE) rate where $\tau_{0}$ is the upper state lifetime, $\tau_{0} \sim 10 \mathrm{~ms}$ [19], and the third term represents the stimulated emission rate to mode group $g$ with photon number $M_{g}$ at the output of EDFA. The subscript ' $g$ ' represents a group of ' $m$ ' very closely spaced cavity modes that have almost the same number of cavity photons $M_{q}$ and same values of $\gamma_{q}$ and $\alpha_{q}$, thus ' $g$ ' replaces the subscript ' $q$ ' and the length-averaged gain coefficient for the $g$ th mode group is expressed as $\overline{g_{g}}$ in (2) where $\overline{g_{g}}=\left(\gamma_{g}+\alpha_{g}\right) \overline{N_{2}}(t)-\alpha_{g}$. The last term on the right-hand side of (2) represents the rate of decay of inversion level due to bidirectional amplified spontaneous emission (ASE). The ASE power (photons/second) emitted for any particular mode group $g$, which is primarily due to the one "extra photon" present for every cavity mode [20], [21], can be written as [22]

$$
P_{\mathrm{ASE}_{g}}=\left(2 \gamma_{g} l \overline{N_{2}}\right) \cdot A_{g} \cdot \Delta \nu_{g}=(2 m / \tau) \cdot \gamma_{g} l \overline{N_{2}} A_{g}
$$

where $\Delta \nu_{g}=m / \tau$ is the bandwidth of the mode group over which the ASE is emitted and $A_{g}=\left(e^{\bar{g}_{g} \cdot l}-1\right) / \bar{g}_{g} \cdot l$ is the ASE amplification factor. The $R_{\mathrm{ASE}}$ term is hence the sum of the ASE over all $\mathrm{g}$ mode groups in both directions less the un-amplified SE term [18] because it is already present in (2)

$$
R_{\mathrm{ASE}}=\sum_{g}(4 m / \tau) \cdot \gamma_{g} l \overline{N_{2}} \cdot\left(A_{g}-1\right) .
$$

The cavity rate equation for all the mode groups can be written as $g$ sets of similar equations

$$
\begin{aligned}
\frac{d M_{g}}{d t} & =\frac{M_{g}}{\tau}\left(G_{f g} \cdot e^{\overline{g_{g}} \cdot l-\alpha_{c}}-1\right)+P_{\mathrm{ASE}_{g}} \\
& =\frac{M_{g}}{\tau}\left(G_{g}-1\right)+P_{\mathrm{ASE}_{g}}
\end{aligned}
$$

where $\alpha_{c}$ is the loss of the cavity external to the EDF but excluding the SLF response (which is represented by $G_{f g}$ for a particular mode group g). $G_{g}$ is the net closed-loop gain for the mode group $g$; which can be expressed as $G_{g}=G_{f g}$. $e^{\overline{g_{g}} \cdot l-\alpha_{c}}$. The parameters $M_{g}, \overline{g_{g}}, \overline{g_{p}}, A_{g}, P_{\mathrm{ASE}_{g}}$ and $R_{\mathrm{ASE}}$ are all function of time $t$ as they are dependent upon $\overline{N_{2}}(t)$ but at steady-state their values become fixed. At steady-state, the favoured mode (say $q_{o}$ ) which is positioned at the centre of any filtered channel (assuming $G_{f q_{o}}=1$ ) and also has slightly more gain than other competitor modes (i.e., the central modes under other filtered channels) starts to win over other modes and saturates the $\overline{N_{2}}$ level until its net closed-loop gain reduces to unity. This essentially means that $\overline{g_{q o}} \cdot l-\alpha_{c}=0$ and the steady-state inversion level $\overline{N_{2 s s}}$ can be easily calculated from this condition as

$$
\overline{N_{2 \mathrm{ss}}}=\left(\alpha_{q o}+\alpha_{c} / l\right) /\left(\alpha_{q o}+\gamma_{q o}\right) .
$$

For single-mode operation of the EDFL, the steady-state power of only one lasing mode is calculated as was done in [18], but for multiwavelength operation the steady-state power of all the mode groups, under selected channels must be known to provide the initial conditions for the start of the phase modulation. This is done by setting the left-hand side of (5) zero and the output power for $g$ th mode group can be evaluated as

$$
\begin{aligned}
P_{g \mathrm{ss}} & =\frac{M_{g \mathrm{ss}}}{\tau}=\frac{P_{\mathrm{ASEss}_{g}}}{\left(1-G_{g}\right)} \\
& =\frac{2 \gamma_{g} l \overline{N_{2 \mathrm{ss}}} A_{g \mathrm{ss}}}{\left(1-G_{f g} \cdot e^{\overline{\overline{g s s}_{g s}} \cdot-\alpha_{c}}\right)} \cdot \Delta \nu_{g} .
\end{aligned}
$$

The steady-state power of the lasing mode can be calculated by setting the left-hand side of (2) to zero, separating out the lasing mode group (for which $\overline{g_{g s \mathrm{~s}}} \cdot l=\overline{g_{q o}} \cdot l=\alpha_{c}$ ) from the summation term and using $P_{g \mathrm{ss}}$ from (7)

$$
\begin{aligned}
P_{q o} & =\left\{P_{p} \cdot\left(1-e^{\overline{g_{p \mathrm{ss}}} l}\right)-(\rho S l) \cdot \frac{\overline{N_{2 \mathrm{ss}}}}{\tau_{0}}\right. \\
& \left.-\sum_{g \neq q o} P_{g \mathrm{ss}} \cdot\left(1-e^{-\overline{g_{g \mathrm{ss}}} l}\right)-R_{A S E s s}\right\} /\left(1-e^{-\alpha_{c}}\right) .
\end{aligned}
$$

It is now clear from (7) that the steady-state ASE power for all the mode groups is dependent upon the transfer function of the periodic filter used in the EDFL cavity. With reasonably uniform gain for the modes within the considered channels, all the mode groups near the transmission peak of the filtered channels would have $G_{g} \approx 1$, so they may have substantial power and stand a good chance to compete with the lasing mode. Only a slight variation in the cavity loss, by changing $\alpha_{c}$ or $K_{f}$, will cause the lasing mode to be switched from one channel to another.

\section{B. Effect of Intra-Cavity Phase Modulation}

A PZT-based sinusoidal phase modulator produces a modulation in the cavity path length. The cavity length $L$ becomes a periodic function of time, $L(t)=L_{o}+\Delta L_{m} \sin \omega_{m} t$ under a typical phase modulation signal $\Phi_{m} \sin \omega_{m} t$, where $L_{o}$ is the fixed length of the EDFL cavity, $\omega_{m}$ is the modulation frequency $(\mathrm{rad} / \mathrm{s})$, and $\Phi_{m}$ is the modulation index (radian). The phase change produced by the PZT is related to the change in the cavity length through the standard equation $\Phi_{m}=2 \pi n \cdot\left(\Delta L_{m} / \lambda_{q}\right)$, 
where $n$ is the effective refractive index and $\lambda_{q}$ is the wavelength of the cavity mode $q$. The change in cavity length will shift the wavelength of the mode to satisfy the phase criterion of the laser cavity: $q \cdot \lambda_{q}=n L$ [20]. Thus, the intra-cavity phase modulation will result in mode-wavelength modulation and the wavelength of a cavity mode will oscillate around its central position as $\lambda_{q}(t)=\lambda_{q}+\lambda_{m} \sin \omega_{m} t$, where $\lambda_{m}=\Delta L_{m} / q$ is the maximum shift in mode-wavelength. This time-varying shift in mode wavelength can be compared with the cavity axial mode spacing $\left(\Delta \lambda_{\mathrm{ax}}=\lambda_{q}^{2} / n L_{o}\right)$ and a relative modulation index (RMI) can be defined as

$$
\mathrm{RMI}=\lambda_{m} / \Delta \lambda_{\mathrm{ax}}=n \cdot \Delta L_{m} / \lambda_{q}=\Phi_{m} / 2 \pi
$$

This relation simply means that when the phase modulation index is $2 \pi$ radians, or when the change in optical path length of the cavity is equal to one wavelength of the cavity mode, all possible cavity modes would then shift on the wavelength axis by an amount equal to $\Delta \lambda_{\mathrm{ax}}$.

Before we consider a full numerical solution to the rate equations under phase modulation, we now give an approximate analytical description of the process to provide physical insight into the multiwavelength operation. With a sinusoidal phase modulation, taking place at a much slower rate than the round trip time of the ring cavity; $T_{m} \gg \tau$ (where $T_{m}$ is the period of the modulation signal), the shift in wavelength of the cavity modes occurs slowly and they see only a slight difference in the absorption and emission coefficients of the EDFA in every round trip. However when a periodic filter, such as the SLF is also present in the laser cavity, the competing modes under selected channels also face a change in the cavity loss when their wavelength position is shifted (Fig. 2) and $G_{f g}$ is now a function of time. As soon as the phase modulation begins at time $t=0$, the loss for the mode already lasing (and other mode groups at the centre of competing channels) increases and its power decreases as described by (5) resulting in an increase in $\overline{N_{2}}$ from its steady-state value according to (2). Since there are many round trips in one modulation cycle, $\overline{N_{2}}(t)$ continues increasing and so does the EDFA gain for all the cavity modes. Thus, the combined effect of the intra-cavity periodic filter and the phase modulation is to make $G_{g}$ and $P_{\mathrm{ASE}_{g}}$ functions of time in (7) and power of every mode group will be a time varying function. With a slowly modulated cavity the circulating power $P_{g}$ will complete a number of round trips within a short fraction of modulation cycle and it can be expressed similar to (7) as

$$
P_{g}(t) \cong \frac{P_{\mathrm{ASE}_{g}}(t)}{\left\{1-G_{g}(t)\right\}}
$$

The dependence of $P_{g}(t)$ on various cavity parameters can be expressed mathematically by differentiating (10)

$$
P_{g}^{\prime}(t) \cong P_{g}(t) \cdot\left\{\frac{G_{g}^{\prime}(t)}{1-G_{g}(t)}+\frac{P_{\mathrm{ASE}_{g}}^{\prime}(t)}{P_{\mathrm{ASE}_{g}}(t)}\right\}
$$

where $G_{g}^{\prime}$ and $P_{\mathrm{ASE}_{g}}^{\prime}$ represent the derivatives of the time varying closed loop gain and the ASE power for the mode $g$. Further expansion of these derivatives using (3) and the definition of $G_{g}$ results in

$$
\begin{aligned}
P_{g}^{\prime}(t) & \cong P_{g}(t) \cdot\left\{X_{g}(t) \cdot{\overline{N_{2}}}^{\prime}(t)+Y_{g}(t) \cdot G_{f g}^{\prime}(t)\right\} \\
& \cong G_{m g}(t) \cdot P_{g}(t)
\end{aligned}
$$

where $\overline{N_{2}}$ and $G_{f g}^{\prime}$ are the derivatives of inversion level and SLF gain respectively. The parameters $X_{g}$ and $Y_{g}$, for a mode group $g$ are given as

$$
\begin{aligned}
X_{g}(t)= & \frac{l\left(\gamma_{g}+\alpha_{g}\right) \cdot G_{g}(t)}{1-G_{g}(t)} \\
& +\frac{\left(\gamma_{g}+\alpha_{g}\right) \cdot\left\{e^{\overline{g_{g}}(t) \cdot l}-A_{g}(t)\right\}}{\overline{g_{g}}(t) \cdot A_{g}(t)}+\frac{1}{\overline{N_{2}}(t)} \\
Y_{g}(t)= & \frac{G_{g}(t)}{G_{f g}(t) \cdot\left\{1-G_{g}(t)\right\}} .
\end{aligned}
$$

Equation (12) describes the change in the optical power of any mode group $g$ and has a solution of the form $P_{g}(t)=P_{g_{0}} \cdot e^{a(t)}$, where $a^{\prime}(t)=G_{m g}(t)$ and $G_{m g}(t)$ represents the time-varying gain factor for a mode group $g$ due to intra-cavity phase modulation. Equation (12) provides an approximate but simple understanding of an EDFL cavity with a periodic filter and low frequency phase modulation. The power of $g$ th mode group can increase exponentially with an instantaneous rate, which is controlled by ${\overline{N_{2}}}^{\prime}$ (rate of change in inversion level) and $G_{f g}^{\prime}$ (rate of change in filter gain).

For mode groups around the transmission peaks of the filter (the favoured mode groups in the multiwavelength operation), which also have a uniform gain from the EDFA the round-trip gain, $G_{g}$ will be close to unity and the first term on the righthand side of (13) will dominate. Also since $G_{f g} \sim 1$ near the filter peaks, we can write the gain factor for the favourite mode groups

$$
G_{m g}(t) \cong\left\{\frac{1}{1-G_{g}(t)}\right\} \cdot\left\{\left(\gamma_{g}+\alpha_{g}\right) l \cdot{\overline{N_{2}}}^{\prime}(t)+G_{f g}^{\prime}(t)\right\} .
$$

Since for a typical flat gain situation $l>20 \mathrm{~m}$, it can be seen from (15) that the effect of ${\overline{N_{2}}}^{\prime}$ is much greater that the $G_{f g}^{\prime}$ for these modes. Also, over the region where the EDFA gain is reasonably flat, these favoured mode groups will experience similar large values of $G_{m g}(t)$, since $\mathrm{G}_{g}$ is similar and close to unity for these mode groups, whereas away from the filter transmission peak $G_{m g}(t)$ will be greatly diminished. In addition to having almost the same magnitude, the phase of $G_{m g}(t)$ will also be the same for all the favoured mode groups. This essentially means that the power of all the favoured mode groups will increase or decrease simultaneously with almost same exponential rate, thus resulting in multiwavelength operation.

Before the start of phase modulation, $G_{f g}^{\prime}$ and ${\overline{N_{2}}}^{\prime}$ are zero and therefore $G_{m g}(t=0)=0$ but as soon as it is turned on, $G_{f g}^{\prime}$ becomes negative for the lasing mode $q_{o}$ and other favoured mode groups at the centre of selected channels. Thus, 
$G_{m g}\left(t=0^{+}\right)<0$ for all such modes and their powers start decreasing exponentially, according to (12), with the rate of decay proportional to their initial power at $t=0$, as also indicated in (12). Hence, in the transient period, the power of the lasing mode, $P_{q o}$, falls much faster than the power of other favoured mode groups. With a slow modulation frequency, $P_{q o}$, along with other mode groups, may then reduce to the ASE level, therefore reducing the total power in the EDFL cavity. As a result ${\overline{N_{2}}}^{\prime}$ will increase according to the atomic rate equation (2) and at some point $G_{m g}(t)$ will reverse sign due to its strong dependence upon ${\overline{N_{2}}}^{\prime}$ from (15), in which case $G_{m g}(t)>0$ for all the mode groups thus providing a simultaneous exponential increase in the power of all mode groups (including mode $q_{o}$ ). However an exponential increase in the total power (from all mode groups) will now start reducing $\overline{N_{2}}(t)$ and the power of all mode groups will decay exponentially when $G_{m g}(t)$ again reverses sign. Hence, a periodic variation in $P_{g}(t)$, mainly controlled by a periodic variation in $\overline{N_{2}}(t)$, will be established for all the favoured mode groups in the EDFL cavity. Although the multiwavelength operation is initiated by $G_{f g}^{\prime}(t)$, it is mainly controlled by ${\overline{N_{2}}}^{\prime}(t)$, provided the modulation frequency is slow enough to allow a variation in $\overline{N_{2}}(t)$ to achieve a sufficient level of multiwavelength gain $G_{m g}(t)$. A relatively higher modulation frequency will not allow $\overline{N_{2}}(t)$ to increase to the desired level and $P_{g}(t)$ will only show a much smaller variation due to the reduced $G_{m g}(t)$. The coupled relationship between $G_{m g}(t)$ and $P_{g}(t)$ can also be expressed by substituting (2) in (12) and rewriting $G_{m g}(t)$ as (16), shown at the bottom of the page. A cross-coupling mechanism among all mode groups can be seen from (16) as $G_{m g}$ for any particular mode depends upon the power of all other mode groups. Since ${\overline{N_{2}}}^{\prime}$ directly depends upon the pump power, $P_{p}$, a higher $G_{f g}^{\prime}$ (due to higher modulation frequency or modulation index) can be compensated by increasing $P_{p}$.

\section{NUMERICAL MODELLING}

As discussed in the last section, under the combined effect of a periodic filter and a phase modulator, the optical power of the cavity modes, $P_{g}(t)$, becomes a time varying function and the time response can be calculated by solving the nonlinear coupled rate (2) and (5). Equations (12) to (16) give an approximate analytical description to provide physical insight into the process but a full numerical solution is required in order to model the effects of various cavity parameters on the multiwavelength operation.

\section{A. Numerical Model}

A MATLAB ${ }^{\circledR}$ program based on (2) and (5) was developed to simulate the operation of the multiwavelength EDFL. The EDF data, provided by the manufacturer, FIBERCORE, was used for the values of the fixed parameters $\rho, \mathrm{S}, \alpha_{p}, \tau_{0}$ and also the absorption $\left(\alpha_{g}\right)$ and emission $\left(\gamma_{g}\right)$ coefficients at wavelength intervals of $0.2 \mathrm{~nm}$. The size of the mode group is defined in the program with a higher resolution than $0.2 \mathrm{~nm}$ and at least 1000 mode groups are chosen between two adjacent channels, so $\Delta \lambda_{g}=s / 1000$ is the spacing between two adjacent mode groups, where ' $s$ ' is the spacing between two adjacent channels of the filter. The values of $\alpha_{g}$ and $\gamma_{g}$ were interpolated at the defined mode groups over a broad spectral range of 1520-1600 $\mathrm{nm}$. The program has two main parts: 1) before the start of phase modulation, the lasing or most favourable wavelength is evaluated, and $\overline{N_{2 s s}}, P_{q o}$ and $P_{g s s}$, for all cavity mode groups within the considered spectrum, are computed, and 2) at $\mathrm{t}=0$, when the phase modulation is switched on, the coupled rate equations are solved for every round trip of the cavity. Since the phase modulation takes place at a much slower rate than the cavity round-trip time $\tau$, a time step equal to $\tau$ is good enough to accurately compute $\overline{N_{2}}(t)$ and $P_{g}(t)$. Although the gain medium, SLF and phase modulator are distributed along the length of the cavity, they are approximately considered as lumped components and the entire cavity length is assumed to exist between the EDFA and the phase modulator. The approximation is good because the modulation is so slow that variation in $G_{f}(\lambda, t)$ and $\overline{N_{2}}(t)$ is quite negligible in one round-trip. After the $i$ th round-trip $(t=i \cdot \tau)$, the rate equations (2) and (5) can be solved numerically as (17) and (18), shown at the bottom of the page, where $\lambda_{g}^{i}=\lambda_{g}^{0}+\lambda_{m} \sin \left(\omega_{m} \cdot i \tau\right)$ sets the wavelength position of the mode group $g$ in the $i$ th round trip and $\lambda_{m}$ is the maximum shift in the position of the mode group due to the phase modulation. Since $\alpha_{g}$ and $\gamma_{g}$ are interpolated at discrete wavelengths of every mode group and their values do not change significantly from one mode group to the next, $\lambda_{m}=\mathrm{RMI} \times \Delta \lambda_{g}$ is set so that a phase modulation index of $2 \pi$ radians can shift the position of one mode group to the position of the next mode group.

Once ${\overline{N_{2}}}^{i}$ is computed, all other time varying parameters such as ${\overline{g_{g}}}^{i},{\overline{g_{p}}}^{i}, R_{\mathrm{ASE}}^{i}, P_{\mathrm{ASE}, g}^{i}$ can also be calculated using

$$
G_{m g}(t)=X_{g}(t) \cdot\left\{\frac{P_{p}\left(1-e^{\overline{g_{p}}(t) \cdot l}\right)-\sum_{g} P_{g}(t) \cdot\left(1-e^{-\overline{g_{g}}(t) \cdot l}\right)-R_{\mathrm{ASE}}(t)}{\rho S l}-\frac{\overline{N_{2}}(t)}{\tau_{0}}\right\}+Y_{g}(t) \cdot G_{f g}^{\prime}(t)
$$

$$
\begin{aligned}
{\overline{N_{2}}}^{i} & ={\overline{N_{2}}}^{i-1}+\tau \cdot\left[\frac{\left\{P_{p} \cdot\left(1-e^{{\overline{g_{p}}}^{i-1} \cdot l}\right)-\sum_{g} P_{g}^{i}\left(1-e^{-{\overline{g_{g}}}^{i-1} \cdot l}\right)-R_{\mathrm{ASE}}^{i-1}\right\}}{\rho S l}-\frac{{\overline{N_{2}}}^{i-1}}{\tau_{0}}\right] \\
P_{g}^{i} & =P_{g}^{i-1} \cdot G_{f g}^{i}\left(\lambda_{g}^{i}\right) \cdot e^{\left({\overline{g_{g}}}^{i-1} \cdot l-\alpha_{c}\right)}+P_{\mathrm{ASE}, g}^{i-1}
\end{aligned}
$$


the standard expressions described in Section II. The condition $i=0$ means the initial condition at $t=0$, when the phase modulation is started in the EDFL cavity, and the initial values for all the time-varying parameters of rate equations are $P_{g \mathrm{ss}}, \overline{N_{2 \mathrm{ss}}}, \overline{g_{p s \mathrm{~s}}}, \overline{g_{g \mathrm{ss}}}, R_{\mathrm{ASEss}}, P_{\mathrm{ASEss}, g}$. In every iteration, $i$, the above set of equations have to be computed for an array of $j$ mode groups simultaneously and $P_{g}^{i}$ will also be an array of power for all these mode groups. When the program runs for a total time of $t=k \cdot \tau$ (with $i=k$ being the last iteration), $P_{g}(t)$ would turn out to be a matrix of size $j \times k$ and provides the time varying power of all $j$ mode groups at the output of the EDFA in units of photons/second; however the output power from the output coupler of the EDFL can easily be calculated in watts as: $\left(h c / \lambda_{g}\right) \cdot \operatorname{R} e^{-\alpha_{o c}} \cdot P_{g}(t)$, where $h$ is the Planck's constant, $c$ is the speed of light, $R$ is the coupler output ratio and $e^{-\alpha_{o c}}$ is the attenuation from the EDFA output to the output coupler.

\section{B. Simulation Results}

The MATLAB ${ }^{\circledR}$ program can simulate the multiwavelength operation of the EDFL shown in Fig. 1 when the pump power $P_{p}$, phase modulation frequency $f_{m}$ and relative modulation index RMI are given as the input parameters. Although we can change the channel spacing of the filter, cavity length, cavity loss, length of EDF and output coupler ratio, the values of these parameters were fixed to match the experimental values. Hence, $s=0.8 \mathrm{~nm}, K_{f}=0.5, L_{o}=42 \mathrm{~m}, l=22 \mathrm{~m}, \alpha_{c}=8.7 \mathrm{~dB}$, $\alpha_{\mathrm{oc}}=1.2 \mathrm{~dB}$, and $R=10 \%$ are set in the program. The size of the mode group is then set as $\Delta \lambda_{g}=0.8 \mathrm{pm}$ with each group consisting of 20 cavity modes. These parameters set the steady state conditions for the EDFL before the start of the phase modulation: the favoured wavelength for lasing is $1563.6 \mathrm{~nm}$ and $\overline{N_{2 \mathrm{ss}}}=0.466$. Fig. 3(a) shows the gain-loss situation for the cavity modes around the lasing mode, while Fig. 3(b) gives the actual power of every mode group when $P_{p}=110 \mathrm{~mW}$. As shown in Fig. 3(a), the cavity loss is minimum $(8.7 \mathrm{~dB})$ at the centre wavelengths of the SLF channels, which appear as inverted peaks in the attenuation plot. As shown in Fig. 3(b), the effect of the periodic filter is very prominent on the EDFL output. Although the closed loop gain is unity for the lasing mode, it is also quite close to unity for the mode groups at the centres of adjacent channels.

Once the phase modulation starts, the coupled equations (17) and (18) have to be solved hundreds of time per modulation cycle and the simulation consumes a lot of time if it solves for all the mode groups $\left(10^{5}\right.$ mode groups in the $1520-1600 \mathrm{~nm}$ range). In order to make the program run efficiently, we only choose 11 mode groups around the peak of the favoured channels. The lasing channel along with 3 channels on either side are considered as the favoured channels. Hence, the coupled rate equations are only solved for 77 mode groups (including the mode already lasing) that are the favoured mode groups for multiwavelength operation because their $G_{g}$ value is very close to unity. The number of selected channels and the number of mode groups per channel can be changed in the program, however it is observed that solving the equations for the remaining high loss mode groups does not affect the behaviour of the selected mode groups but only increases the simulation time unnecessarily. To further reduce the complexity and time of the simulations,

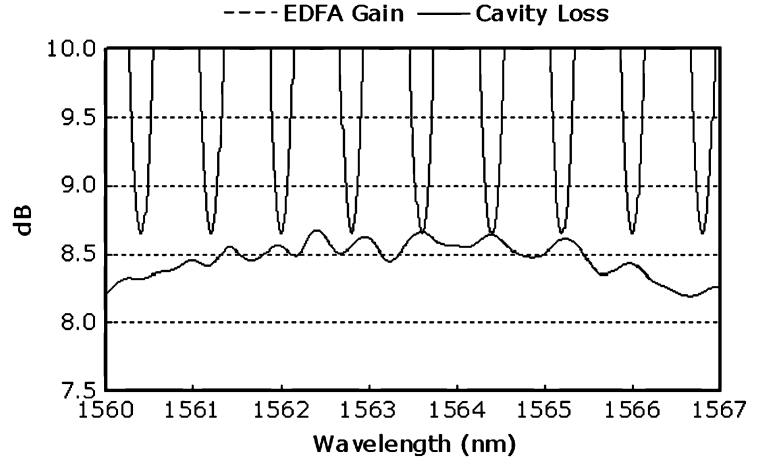

(a)

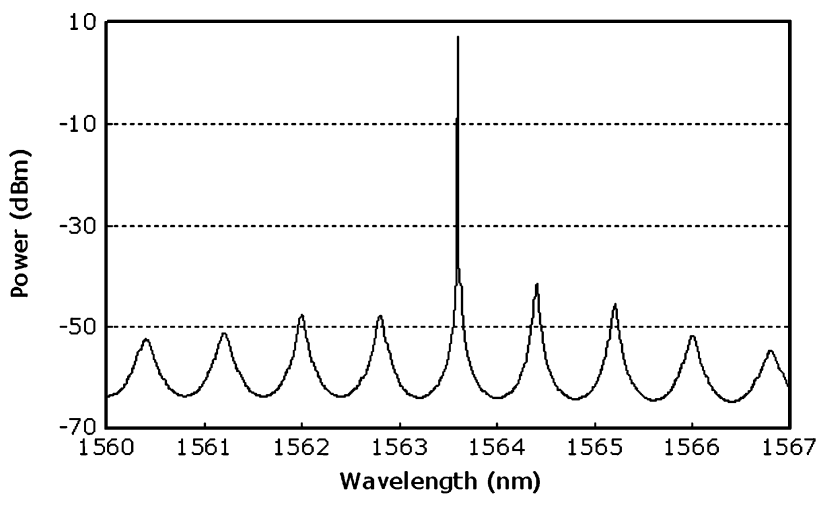

(b)

Fig. 3. Simulation results with phase modulator OFF; $P_{p}=110 \mathrm{~mW}, s=$ $0.8 \mathrm{~nm}$. (a) Gain and loss distribution for competing channels of periodic filter. (b) Computed output spectrum showing power in lasing mode and in adjacent channels.

two more approximations are made: 1) for any particular mode group $g$ the EDF parameters $\alpha_{g}$ and $\gamma_{g}$ are assumed constant over the entire modulation period i.e., the wavelength shift from the phase modulation is small; and 2) $R_{\mathrm{ASE}}$ remains unchanged with time. These assumptions do not affect the accuracy of the numerical results as long as the RMI $\leq 5$ and the variation in $\overline{N_{2 \mathrm{ss}}}$ is small. Since $P_{g}(t)$ for all the mode groups vary with time simultaneously and the 11 mode groups under any filter channel are very closely spaced, the powers of all mode groups under a channel are added to give the channel power, and the power of all channels can be added to get the total output power from the EDFL. The simulation runs typically for 15 modulation cycles.

For our EDFL, the round-trip frequency is $5 \mathrm{MHz}$ and with $P_{p}=110 \mathrm{~mW}$, the relaxation oscillation frequency [18] is computed to be $28 \mathrm{kHz}$ approximately. Simulations were carried out at various low modulation frequencies $(1-30 \mathrm{kHz})$ and then both $P_{p}$ and RMI were also changed at these frequencies to understand their effects on $P_{g}(t)$ of the selected mode groups. Fig. 4 shows the results of the simulation when $f_{m}=17 \mathrm{kHz}$, $\mathrm{RMI}=1$ and $P_{p}=110 \mathrm{~mW}$. It can be seen that as soon as the phase modulation starts $P_{g}(t)$ starts decreasing for all the mode groups and $\overline{N_{2}}(t)$ starts increasing above its $\overline{N_{2 s s}}$ level. This results in an increase in the EDFA gain and $P_{g}(t)$ starts increasing again. As can be seen in Fig. 4(a), after a few cycles a regular periodic variation in $\overline{N_{2}}$ is achieved and the periodic variations in $\overline{N_{2}}$ and $G_{f g}$ result in periodic and simultaneous power changes in all mode groups. The 3-D plot, shown in Fig. 4(b), presents 


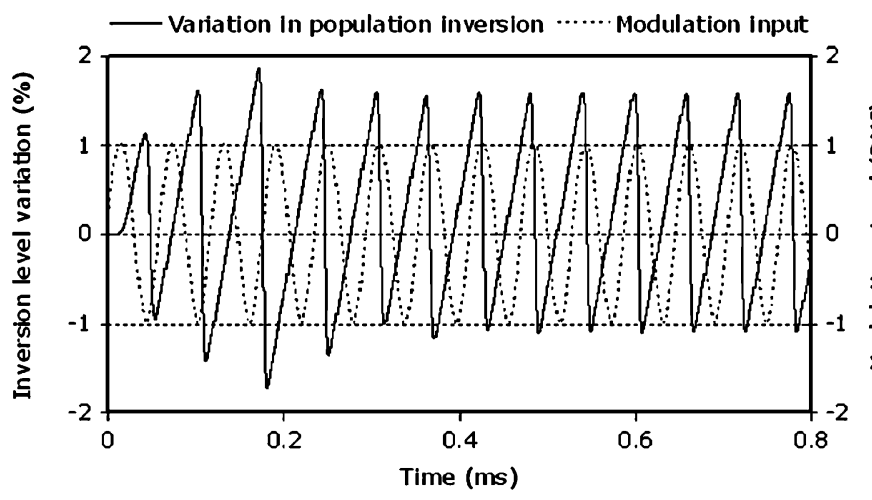

(a)

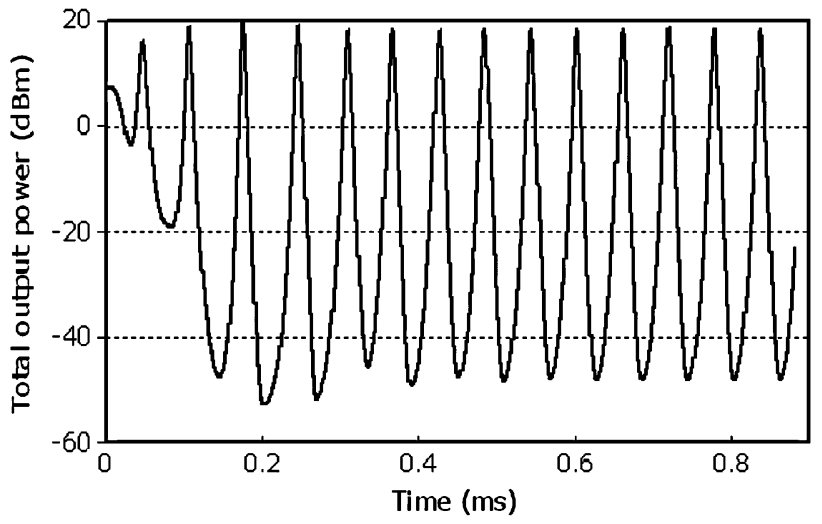

(c)

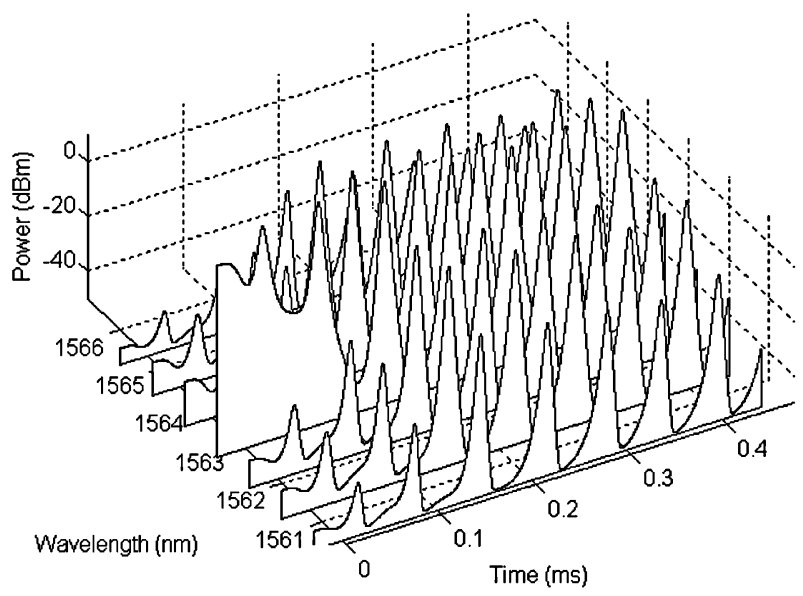

(b)

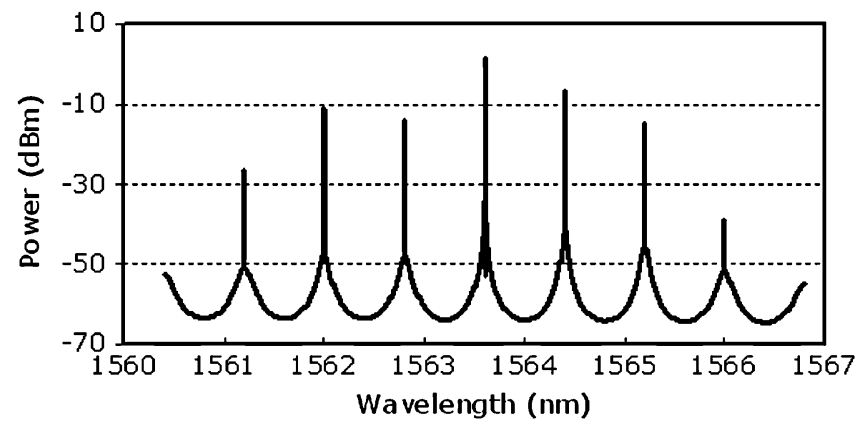

(d)

Fig. 4. Simulation results with phase modulator ON; $f_{m}=17 \mathrm{kHz}, \mathrm{RMI}=1$. (a) Variation in $\overline{N_{2 \mathrm{ss}}}$ and modulation signal. (b) Output power for selected channels. (c) Total output power of EDFL. (d) Multiwavelength spectrum.

the instantaneous power of 7 channels (whose round-trip gain is close to unity). The centre wavelengths of the channels are shown on the $y$-axis, their optical power on the $\mathrm{z}$ axis and time on the $\mathrm{x}$-axis. It can be seen that after $\sim 0.1 \mathrm{~ms}$ the power of every channel decreases and increases exponentially and acquires the shape of a pulse. Another important observation, from Fig. 4(b), is that the pulsed outputs of the channels appear simultaneously and periodically at a rate equal to the modulation frequency. Since the time-domain response for all channels is perfectly simultaneous, their instantaneous powers can simply be added to give the total power of the multiwavelength EDFL. The behaviour of the total output power, shown in Fig. 4(c), is therefore exactly the same as the behaviour of every individual channel. The duty cycle of the pulse is $15.2 \%$ and the peak total power is $67.2 \mathrm{~mW}$, which is almost 13 times the $\mathrm{CW}$ power of the EDFL before the start of phase modulation. In order to see the intensity of individual channels, an optical spectrum is plotted in Fig. 4(d) which is based on the average channel power per modulation cycle. A comparison of Fig. 4(d) with Fig. 3(a) shows that the power distribution among the multiwavelength channels reflect the EDFA gain profile (specifically the EDFA round-trip gain $G_{g}$ ), and a more uniform multiwavelength spectrum can be achieved with better gain flattening of the EDFA.

When $f_{m}$ was increased above $17 \mathrm{kHz}$, the power pulses start losing their sharpness and the peak-to-peak amplitude of the pulse reduces and becomes irregular. Fig. 5(a) shows such a behaviour when $f_{m}=22 \mathrm{kHz}$. The multiwavelength spectrum can still be observed in Fig. 5(b) because the mode groups under every channel still get sufficient power variation; but the power in the side channels is now much less when compared to Fig. 4(d). When $f_{m}$ is further increased to $30 \mathrm{kHz}$, the peak-topeak variation in pulses was further reduced to $20 \mathrm{dBm}$, the multiwavelength coupling becomes almost absent and the optical spectrum is similar to Fig. 3(b). Further increase in the modulation frequency results in only a small ripple in the channel powers and the EDFL output can be considered as essentially $\mathrm{CW}$, as it was before the start of phase modulation. A similar trend was observed when the RMI was increased, keeping $f_{m}$ fixed at $17 \mathrm{kHz}$. The behaviour can be explained qualitatively from (15) because an increase in $f_{m}$ or RMI makes $G_{f g}$ change more quickly but $\overline{N_{2}}(t)$ cannot increase by a significant amount in a relatively shorter span of time. As a result $P_{g}$ also varies quickly but the multiwavelength gain factor $G_{m g}$ is not sufficient to form a pulse-like shape. Nevertheless if the pump power is increased to increase ${\overline{N_{2}}}^{\prime}$ the required $G_{m g}$ can be restored and the regular pulsed output could be achieved at a higher frequency or RMI. This was confirmed through simulations and stable multiwavelength operation was achieved at RMI $=2, f_{m}=19.5 \mathrm{kHz}, P_{p}=150 \mathrm{~mW}$ and also at $\mathrm{RMI}=2, f_{m}=22 \mathrm{kHz}, P_{p}=190 \mathrm{~mW}$. The time and fre- 


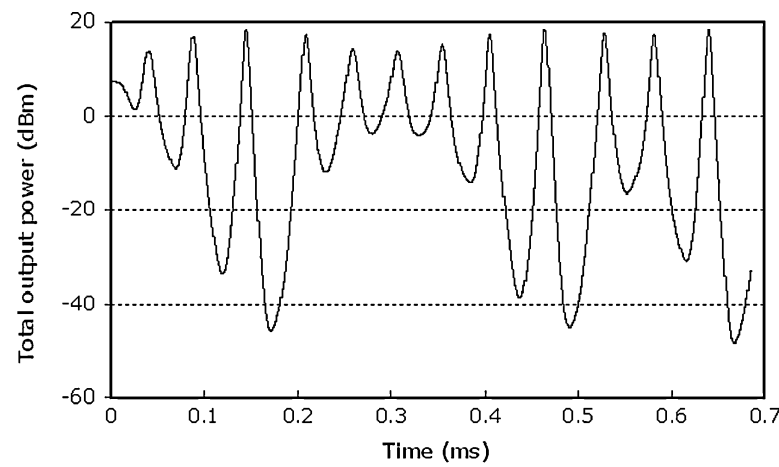

(a)

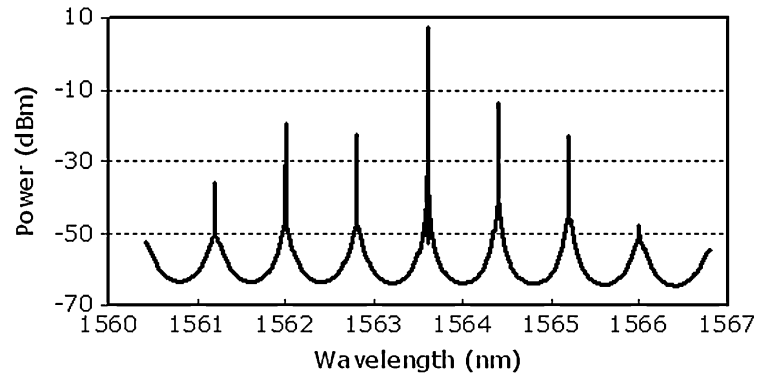

(b)

Fig. 5. Simulation results at $f_{m}=22 \mathrm{kHz}$. (a) Total output power of EDFL. (b) Multiwavelength spectrum.

quency domain behaviour for these conditions was quite similar to the results shown in Fig. 4.

Correspondingly, when $f_{m}$ was reduced below $17 \mathrm{kHz}$, keeping RMI $=1$ and $P_{p}=110 \mathrm{~mW}$, both time and frequency domain outputs remained stable; the frequency of the pulses changed with $f_{m}$ and it was observed that the pulses became sharper, with their peak power increasing and duty cycle decreasing gradually. So at $10 \mathrm{kHz}$ the peak EDFL power and the duty cycle were $186 \mathrm{~mW}$ and $4.9 \%$ respectively and the power of individual channels had also increased. Below $10 \mathrm{kHz}$, the time domain response became irregular and high frequency power fluctuations occurred with varying amplitude; however the multiwavelength spectrum remained similar to Fig. 4(d) because the mode groups in all channels still get sufficient gain. This can be understood by noting that when $G_{f g}$ varies at a slow rate, $\overline{N_{2}}(t)$ can get a large variation within the relatively longer modulation period resulting in a high increase in $P_{g}$. Such an increase in power of all modes will reduce their respective gain $G_{m g}(16)$ even before the completion of one modulation cycle and therefore faster power variations appear. However when ${\overline{N_{2}}}^{\prime}$ is reduced by reducing $P_{p}$, a periodic pulsed pattern can again be obtained. Thus, stable multiwavelength operation was achieved at $f_{m}=9 \mathrm{kHz}, P_{p}=90 \mathrm{~mW}$ and $f_{m}=5 \mathrm{kHz}$, $P_{p}=50 \mathrm{~mW}$, keeping RMI $=1$ in both cases.

In summary, the simulations show that the combined effect of intra-cavity phase modulation and periodic filtering produces simultaneous pulses of power for every channel and the EDFL output is not CW. Stable operation is achieved when $G_{f g}^{\prime}$ and ${\overline{N_{2}}}^{\prime}$ are controlled in such a way that regular pulses appear at a frequency equal to $f_{m}$. A multiwavelength spectrum, with a stable time-domain response for every channel, can be achieved at different combinations of $P_{p}, f_{m}$, and RMI, as long as $f_{m}$ is

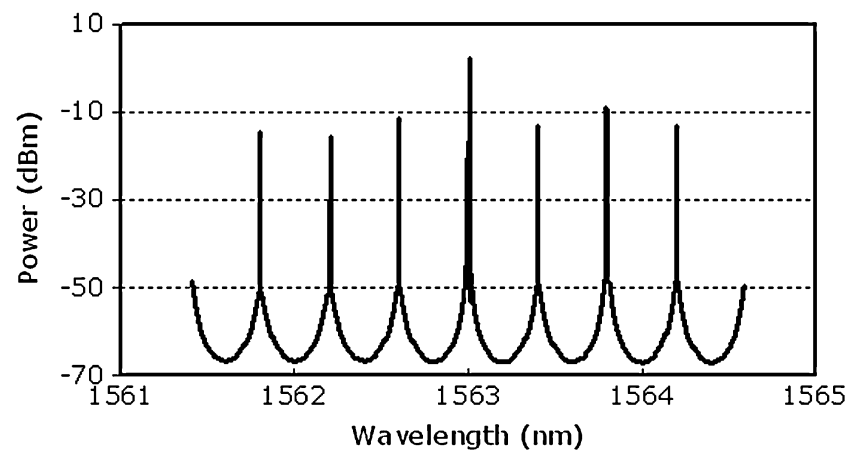

Fig. 6. Simulation results for spectrum with reduced channel spacing $s=$ $0.4 \mathrm{~nm}$.

considerably smaller than the relaxation oscillation frequency of the EDFL. As observed in the simulations, slight variations in these parameters do not affect the performance of the multiwavelength EDFL. It is also shown that multiwavelength channels can have a reasonably uniform power level if the EDFA gain is almost flat for these channels. So when the channel spacing $s$ is reduced to bring the channels closer and hence experience approximately similar gain, the uniformity of power improves for seven selected channels (Fig. 6). Gain flattening over a certain range and hence a uniform multiwavelength spectrum can also be achieved by increasing the EDF length and/or reducing the cavity loss to achieve a lower average inversion level. The multiwavelength EDFL appears to be more stable than its corresponding single-wavelength EDFL (without periodic filtering and phase modulation) because it lases at all favoured modes, selected by the filter, and mode hopping cannot take place. So the stability of lasing wavelengths only depends on the stability of the filter response and not on the entire cavity length.

\section{EXPERIMENTAL Study OF MULTiwaVELENGTH EDFL}

An experimental multiwavelength EDFL system was set up using the design shown in Fig. 1. The SLF was constructed by connecting the two ends of a 50/50 single mode coupler to $7.2 \mathrm{~m}$ of polarization maintaining fiber (PMF), whose birefringence was $4.2 \times 10^{-4}$, to produce a channel spacing $s \sim 0.8 \mathrm{~nm}$. In order to control $K_{f}$ of the SLF, a polarization controller (PC) was added at one end of the SLF. The characteristics of the PZT based phase modulator were independently measured by placing it in one arm of a Mach-Zehnder interferometer and detecting the harmonics of the phase modulated signal on a lock-in amplifier. The measured frequency response of the phase modulator is shown in Fig. 7 and its peak gain was $15.7 \mathrm{rad} / \mathrm{V}_{\mathrm{p}-\mathrm{p}}$ at the resonance frequency $(19.5 \mathrm{kHz})$ of the PZT. Away from the resonance frequency, the gain of the phase modulator drops off sharply and its value is reduced to $1.8 \mathrm{rad} / \mathrm{V}_{\mathrm{p}-\mathrm{p}}$ at 17 or $22 \mathrm{kHz}$. For the EDFL the phase modulator was driven by a variable frequency sine-wave signal, whose amplitude could be varied from 0 to $10 \mathrm{~V}_{\mathrm{p}-\mathrm{p}}$. The input signal was dc shifted to drive the PZT with single polarity. In order to get a reasonably flat gain from the amplifier in the ring laser cavity, two independent but similar EDFA units were cascaded. Each EDFA unit has approximately $11 \mathrm{~m}$ of EDF with its own isolators and WDM couplers. Both EDFA units have a separate pump source $(980 \mathrm{~nm})$, whose 


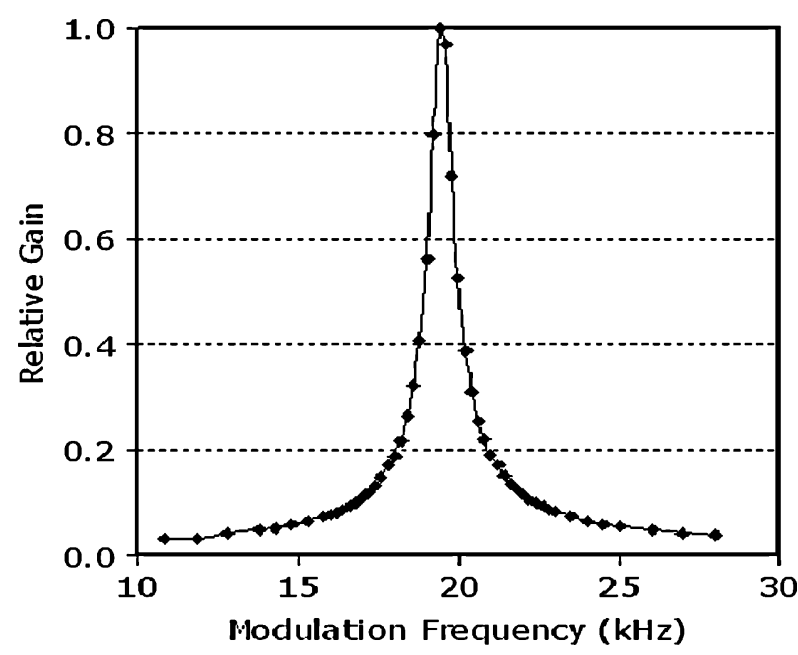

Fig. 7. Measured frequency response of the phase modulator.

power can be controlled from 0 to $70 \mathrm{~mW}$. A $10 \%$ tap coupler was used to take the output power from the EDFL, which was further divided by another 50/50 coupler (not shown in Fig. 1) in order to observe both time and frequency domain behaviour of the multiwavelength EDFL simultaneously.

Fig. 8 shows the measured optical spectrum of the multiwavelength EDFL when the phase modulator is switched off. The effect of the SLF on the background ASE can be clearly seen. Although the EDFL is lasing at one wavelength channel $(1561 \mathrm{~nm}$ approximately), a slight adjustment of the PC can easily switch the lasing wavelength to any of the adjacent channels. Hence, with the help of a PC inside the SLF the favoured channel can be selected for lasing. Another PC (not shown in Fig. 1) was initially used in the main EDFL cavity to see if it could bring any further control on the output. However, irrespective of its location in the cavity, the second PC did not affect the laser performance at all and the EDFL did not show any polarization dependence. When the phase modulator was switched on, the multiwavelength operation could only be measured over a limited PZT frequency range $\left(f_{m \sim 17-22 \mathrm{kHz}}\right)$ due to the narrow bandwidth of the phase modulator (Fig. 7). Initially $f_{m}$ was set at $19.5 \mathrm{kHz}$, as the gain of the phase modulator is maximum at this frequency and an input signal of $V_{m}=0.4 \mathrm{~V}_{\mathrm{p}-\mathrm{p}}$ was applied, corresponding to $2 \pi$ radians and RMI $\sim 1$. When the pump power of each EDFA was increased to $35 \mathrm{~mW}\left(P_{p}=\right.$ $2 \times 35=70 \mathrm{~mW}$ ), the power in the channels adjacent to the lasing channel began to increase and the $\mathrm{CW}$ power changed into pulses with unstable frequency and amplitude. Increasing $P_{p}$ to $80 \mathrm{~mW}$ and RMI to 2 , the multiwavelength spectrum became reasonably stable and the power in all channels became more stable. Fig. 9(a) shows the stable multiwavelength spectrum where $P_{p}=2 \times 45=90 \mathrm{~mW}, f_{m}=19.5 \mathrm{kHz}$ and $V_{m}=$ $1.0 \mathrm{~V}_{\mathrm{p}-\mathrm{p}}(\mathrm{RMI} \sim 2.5)$. Fig. 9 (b) shows the corresponding time domain response where it can be seen that the output power consists of periodic pulses with the same frequency as $f_{m}$. Although Fig. 9(b) only presents the measured values over a short time span to clearly show the relation between the modulation input and the EDFL output, the pulsed response was reasonably stable even after a few hours. The peak power was $5.9 \mathrm{~mW}$

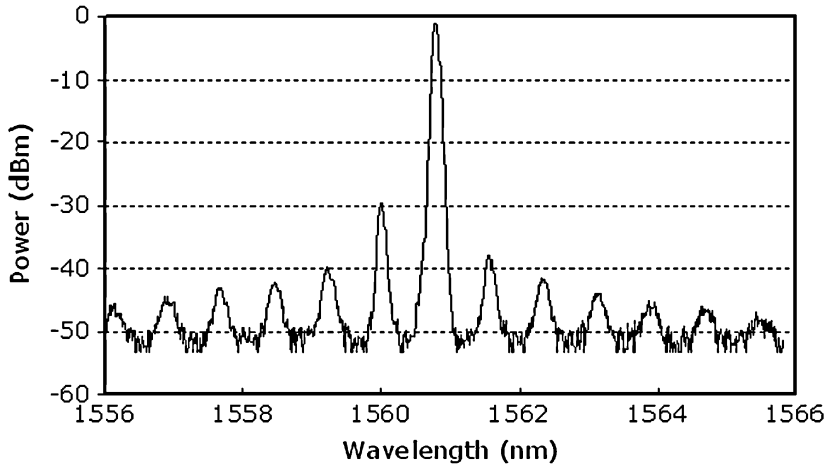

Fig. 8. Experimentally measured spectrum with phase modulator OFF.

and the duty cycle of the pulse was measured as $11 \%$ approximately. When the total pump power was further increased to $100 \mathrm{~mW}$, the peak power was increased to $7.7 \mathrm{~mW}$ and duty cycle reduced to almost $9.5 \%$. Increasing $V_{m}$ in small steps did not affect the output significantly; however when RMI $>5$, the output pulses became unstable with varying amplitude and frequency. In order to observe the effect of modulation frequency, $f_{m}$ was reduced to $17 \mathrm{kHz}$ and a stable multiwavelength spectrum with pulsed output in the time domain was achieved with $P_{p}=2 \times 35=70 \mathrm{~mW}$ and $V_{m}=7.0 \mathrm{~V}_{\mathrm{p}-\mathrm{p}}(\mathrm{RMI} \sim 2)$. However as soon as $P_{p}>80 \mathrm{~mW}$ the multiwavelength operation again becomes unstable. Similarly when $f_{m}$ was increased to $22 \mathrm{kHz}$ with $V_{m}=7.0 \mathrm{~V}_{\mathrm{p}-\mathrm{p}}(\mathrm{RMI} \sim 2)$, the total pump power had to be increased to $110 \mathrm{~mW}$ to achieve a stable spectrum with pulsed output at $22 \mathrm{kHz}$. Again when $P_{p}>120 \mathrm{~mW}$ multiwavelength operation ceased to exist. At 17 or $22 \mathrm{kHz}, V_{m}$ could only be increased to $10 \mathrm{~V}_{\mathrm{p}-\mathrm{p}}$ (the maximum limit of the signal generator) and this small change in RMI did not affect the multiwavelength operation. Note that in all cases, when the phase modulation is switched off, the spectrum returns to that of Fig. 8 with output power of $0.8 \mathrm{~mW}(\mathrm{CW})$ for $P_{p}=90 \mathrm{~mW}$.

Although the experiments could not be carried out over a big range of $f_{m}, P_{p}$ and RMI, the measured results have validated the simulation results based on the numerical model. In all these experiments it was found that a stable multiwavelength spectrum is linked with a stable and periodic pulse train in the time domain at a repetition rate equal to modulation frequency. Stable multiwavelength operation of the EDFL can be achieved at different combinations of $f_{m}, P_{p}$ and RMI and it was observed that as the pulse gets sharper the spectrum becomes more stable. The inter-relationship of these parameters, as found through the simulations, is also confirmed experimentally when it was observed that when $f_{m}$ or RMI are increased then $P_{p}$ has also to be increased to get a multiwavelength output. However the experimental value of $P_{p}$, to get stable multiwavelength operation at a given $f_{m}$ is lower than the corresponding value in the simulations. The possible reason for this difference is that the cascaded EDFA units, with distributed pump sources, need less pump power to attain the required variation in $\overline{N_{2}}(t)$. Similarly, stable multiwavelength operation was achieved in the simulations when RMI $=1$, whereas in experiments a higher value of RMI was required. The simple reason for such a variation is that the size of mode group in 


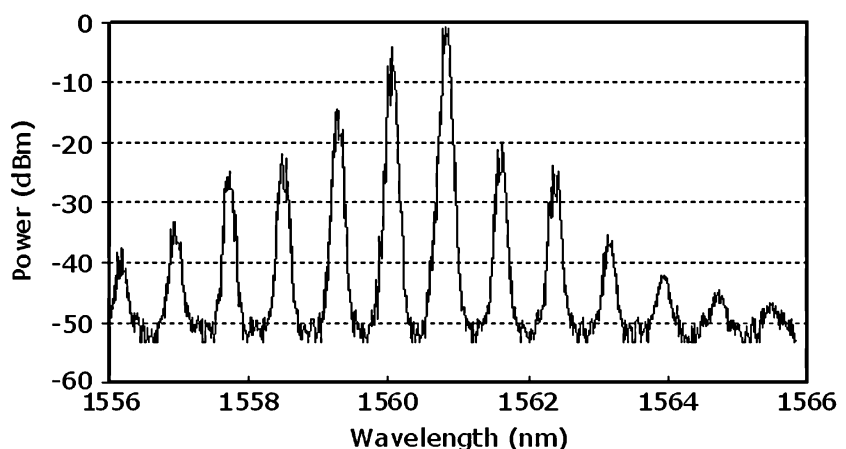

(a)

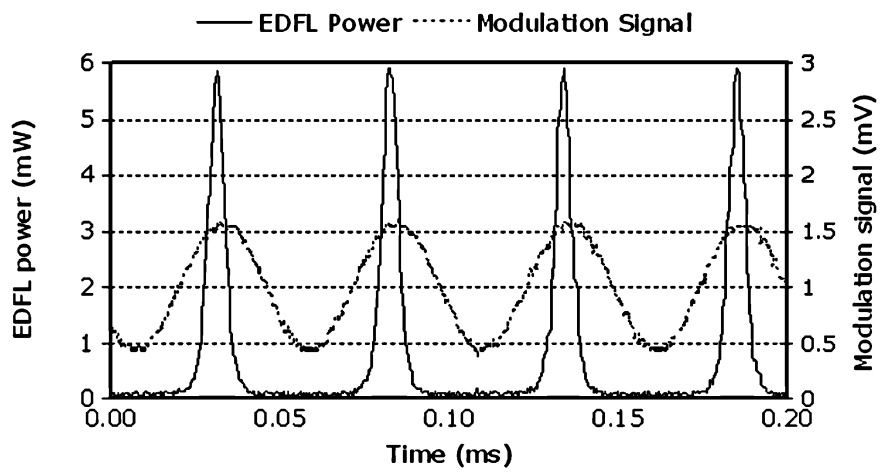

(b)

Fig. 9. Multiwavelength EDFL measurements with phase modulator ON (a) Optical spectrum. (b) Time-domain response.

the simulations is big enough to produce a sufficient change in $G_{f g}$ with RMI $=1$ only. Another very important observation is the role of the PC inside the SLF; a fine adjustment of the PC can help in fine tuning of the multiwavelength spectrum and enhance the uniformity of power in adjacent channels. However a very coarse adjustment of the PC can increase the cavity loss, making the EDFA gain nonuniform and thus stopping the multiwavelength action.

\section{Potential Sensor ApPlications}

Development of multiwavelength fiber lasers in recent years has mainly focused on their application in optical communications where they are useful for DWDM communication, component testing and frequency referencing [11]. However the multiwavelength EDFL presented in this paper can offer some important advantages in comparison to other multiwavelength lasers due to its flexible external cavity design. These include (i) the length of the gain medium (EDF) can be adjusted to control the flatness of the multiwavelength spectrum, (ii) a multiwavelength spectrum can be achieved anywhere within the wide bandwidth of the EDFA by controlling the cavity loss, (iii) periodic filters of different designs, with tuneable channel position and spacing, can be inserted in the cavity to obtain a desired spectrum, (iv) the power in every channel is relatively higher due to the pulsed output. Simultaneous pulses for multiple channels may have potential applications in the field of multipoint distributed fiber optic sensing, especially when sensors are multiplexed through WDM and TDM topologies [23]. A set of fiber Bragg grating (FBG) sensors, each with slightly different wavelengths, can be interrogated simultaneously by the multiwavelength EDFL

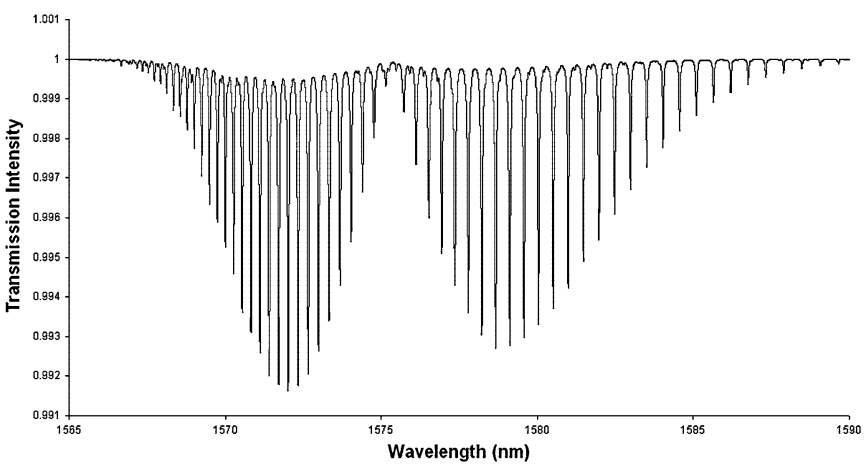

Fig. 10. Absorption spectrum of $\mathrm{CO}_{2}$ at 1.013 bar computed from HITRAN database.

when it is designed using similar FBGs as the multiwavelength filter in the laser cavity. Since this EDFL produces a pulsed output, with a repetition rate of a few $\mathrm{kHz}$, spatially distributed sets of such FBG sensors could be interrogated on a single fiber by the TDM method and use of a single wavelength detection system at the receiving end. In comparison to a broadband $\mathrm{CW}$ light source, the multiwavelength EDFL not only gives higher power per channel but also its low frequency pulsed output is advantageous as the number of multiplexed sensors can be increased, depending upon the width and frequency of the pulses. This is particularly important, for example, in the use of FBG sensors for damage prognosis where monitoring of large structures is required.

Other potential applications for multiwavelength EDFLs are in gas sensing and spectroscopy. Various gases of environmental importance, such as $\mathrm{CO}_{2}$, have multiple rotational absorption lines in near-IR band. Fig. 10 shows the P- and $\mathrm{R}$ - absorption bands of $\mathrm{CO}_{2}$ around 1572 and $1578.6 \mathrm{~nm}$; in each band the absorption lines are approximately uniformly spaced by $\sim 0.3 \mathrm{~nm} .{ }^{1}$ A multiwavelength EDFL could be carefully designed for these bands by using a periodic SLF, whose channels have the same uniform spacing, with the centre wavelengths matched to the absorption line positions. Such a multiwavelength source is particularly useful in detecting $\mathrm{CO}_{2}$ in the presence of other interfering gases which may have one or more absorption lines coinciding with those of $\mathrm{CO}_{2}$ but with different line spacing. Single wavelength fiber lasers have also been considered for intra-cavity laser absorption spectroscopy (ICLAS) [24]. An intra-cavity multiwavelength gas sensor may be of particular interest for future research where the gas cell is placed within the cavity and the gas absorption spectrum forms an intra-cavity periodic filter, with the multiwavelength spectrum of the EDFL corresponding to the transmission maxima between the absorption lines. A change in the gas concentration or pressure will change the width of absorption lines and hence $G_{f g}^{\prime}$ will vary for fixed $f_{m}$ and RMI. Thus, it may be possible to find a correlation between the parameters of multiwavelength operation and the gas properties.

\section{CONCLUSION}

We have performed a detailed theoretical as well as experimental investigation of the dynamics of a multiwavelength

\footnotetext{
${ }^{1}$ HITRAN Database, 2004.
} 
EDFL using intra-cavity periodic filtering and phase modulation. It has been shown mathematically that, with a low frequency phase modulation, the gain of the favoured cavity modes becomes a time-varying function which is strongly dependent on the pump power, modulation index and frequency. A numerical model has been developed in MATLAB ${ }^{\circledR}$ that numerically solves the atomic and cavity rate equations for the EDFL under a given set of parameters for the cavity, filter and phase modulator. Simulations have shown that the output of the multiwavelength EDFL is not $\mathrm{CW}$ but takes the shape of a well-defined pulse repeating at a rate equal to the modulation frequency when the pump power, modulation frequency and index are carefully controlled. Experimental measurements, in both time and frequency domains, made on an EDFL constructed using a SLF and a PZT-based phase modulator have confirmed the mathematical analysis and numerical simulations. It was observed that the multiwavelength spectrum is stable whenever the laser output pulses are stable in the time domain, at the modulation frequency and such a stable output can be achieved with different combinations of pump power, modulation index and frequency, provided the modulation frequency is less than the relaxation oscillation frequency. The all-fiber multiwavelength laser reported here, with a simple means for producing multiwavelength lasing, has potential application as a light source in fiber gas sensing and structural health monitoring using FBG sensors. The production of simultaneous and relatively high power pulses for all the lasing wavelengths provides additional benefits such as potential use in time division multiplexing of spatially distributed sensors. The design is also flexible; channel wavelengths, channel spacing, pulse frequency and pulse power can be easily adjusted by choice of components and cavity parameters.

\section{REFERENCES}

[1] N. Park and P. F. Wysocki, "24-Line multiwavelength Operation of erbium-doped fiber-ring laser," IEEE Photon. Technol. Lett., vol. 8, no. 11, pp. 1459-1461, Nov. 1996.

[2] S. Yamashita and K. Hotate, "Multiwavelength erbium-doped fiber laser using intracavity etalon and cooled by liquid nitrogen," Electron. Lett., vol. 32, no. 14, pp. 1298-1299, Jul. 1996.

[3] J. Chow, G. Town, B. Eggleton, M. Ibsen, K. Sugden, and I. Bennion, "Multiwavelength generation in an erbium-doped fiber laser using in-fiber comb filters," IEEE Photon. Technol. Lett., vol. 8, no. 1, pp. 60-62, Jan. 1996.

[4] O. Graydon, W. H. Loh, R. I. Laming, and L. Dong, "Triple-frequency operation of an er-doped twincore fiber loop laser," IEEE Photon. Technol. Lett., vol. 8, no. 1, pp. 63-65, Jan. 1996.

[5] D. R. Chen, Z. W. Yu, S. Qin, and S. L. He, "Switchable dual-wavelength raman erbium-doped fiber laser," Electron. Lett., vol. 42, no. 4, pp. 202-204, Feb. 2006.

[6] M. H. Al-Mansoori, F. R. M. Adikan, A. W. Naji, M. K. Abdullah, and M. A. Mahdi, "Multiwavelength fabry-perot brillouin-erbium fiber laser," Int. J. Light Electron Opt., vol. 117, no. 3, pp. 128-130, Mar. 2006.

[7] Y. J. Song, L. Zhan, J. H. Ji, Y. Su, Q. H. Ye, and Y. X. Xia, "Selfseeded multiwavelength brillouin-erbium fiber laser," Opt. Lett., vol. 30, no. 5, pp. 486-488, Mar. 2005.

[8] Y. G. Han, G. Kim, J. H. Lee, S. H. Kim, and S. B. Lee, "Lasing wavelength and switchable multiwavelength fiber laser from 1510 to 1620 nm," IEEE Photon. Technol. Lett., vol. 17, no. 5, pp. 989-991, May 2005.

[9] X. Yang, X. Dong, S. Zhang, F. Lu, X. Zhou, and C. Lu, "Multiwavelength erbium-doped fiber laser with $0.8-\mathrm{nm}$ spacing using sampled bragg grating and photonic crystal fiber," IEEE Photon. Technol. Lett., vol. 17 , no. 12 , pp. 2538-2540, Dec. 2005.
[10] A. Zhang, H. Liu, M. S. Demokan, and H. Y. Tam, "Stable and broad bandwidth multiwavelength fiber ring laser incorporating a highly nonlinear photonic crystal fiber," IEEE Photon. Technol. Lett., vol. 17, no. 12, pp. 2535-2537, Dec. 2005.

[11] A. Bellemare, M. Karasek, M. Rochette, S. Larochelle, and M. Tetu, "Room temperature multifrequency erbium-doped fiber lasers anchored on the ITU frequency grid," J. Lightw. Technol., vol. 18, no. 6, pp. 825-831, Jun. 2000.

[12] J. Yao, J. Yao, Z. Deng, and J. Liu, "Multiwavelength erbium-doped fiber ring laser incorporating an SOA-based phase modulator," IEEE Photon. Technol. Lett., vol. 17, no. 4, pp. 756-758, Apr. 2005.

[13] F. Ahmed, N. Kishi, and T. Miki, "Multiwavelength erbium-doped fiber fabry-perot laser and its uniform spectral lines power operation," IEEE Photon. Technol. Lett., vol. 17, no. 4, pp. 753-755, Apr. 2005.

[14] K. Zhou, D. Zhou, F. Dong, and N. Q. Ngo, "Room-temperature multiwavelength erbium doped fiber ring laser employing sinusoidal phasemodulation feedback," Opt. Lett., vol. 28, no. 11, pp. 893-895, Jun. 2003.

[15] M. A. Mirza and G. Stewart, "Design of a multiwavelength fiber laser using an intra-cavity phase modulator and Sagnac loop filter for sensor applications," in Proc. SPIE, 3rd Eur. Workshop Optical Fiber Sens., Napoli, Italy, Jul. 4-6, 2007, vol. 6619, pp. K1-K4.

[16] Y. Sun, J. L. Zyskind, and A. K. Srivastava, "Average inversion level, modelling and physics of erbium-doped fiber amplifiers," IEEE J. Sel. Topics Quantum Electron., vol. 3, no. 4, pp. 991-1007, Aug. 1997.

[17] D. B. Mortimore, "Fiber loop reflectors," J. Lightw. Technol., vol. 6, no. 7, pp. 1217-1224, Jul. 1988

[18] G. Stewart, G. Whitenett, K. Vijayraghavan, and S. Sridaran, "Investigation of the dynamic response of Erbium fiber lasers with potential application for sensors," J. Lightw. Technol., vol. 25, no. 7, pp. 1786-1796, Jul. 2007.

[19] , M. J. F. Digonnet, Ed., Rare-Earth-Doped Fiber Lasers and Amplifiers. New York: Marcel Dekker, 2001.

[20] A. E. Siegman, Lasers.. Mill Valley, CA: University Science Books, 1986, pp. 502-503, 980-982,

[21] P. C. Becker, N. A. Olsson, and R. J. Simpson, Erbium Doped Fiber Amplifiers.. New York: Academic, 1999, pp. 147-157.

[22] C. R. Giles and E. Desurvire, "Modeling erbium-doped fiber amplifiers," J. Lightw. Technol., vol. 9, no. 2, pp. 271-283, Feb. 1991.

[23] A. D. Kersey, M. A. Davis, H. J. Patrick, M. LeBlanc, K. P. Koo, C. G. Askins, M. A. Putnam, and E. J. Friebele, "Fiber Grating Sensors," $J$. Lightw. Technol., vol. 15, no. 8, pp. 1442-1463, Aug. 1997.

[24] G. Stewart, P. Shields, and B. Culshaw, "Development of fiber laser systems for ring-down and intra-cavity gas spectroscopy in the nearIR," Meas. Sci. Technol., vol. 15, no. 8, pp. 1621-1628, Aug. 2004.

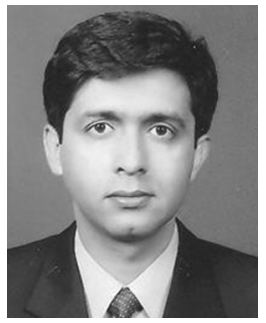

Mohammad Aleem Mirza received the B.Sc. degree (hons.) in electrical engineering from The University of Engineering and Technology, Lahore, Pakistan in 1993, and the M.Sc. degree in electrical engineering from The Engineering University, Taxila, Pakistan, in 2004, with research emphasis on PMD measurement. $\mathrm{He}$ is currently working toward the Ph.D. degree in the electronic and electrical engineering department of Strathclyde University, Glasgow, U.K.

He has gained industrial work experience in the field of fiber optic communication. His present research interest is in the design of multiwavelength erbium-doped fiber lasers. $\mathrm{He}$ is author or coauthor of four conference papers.

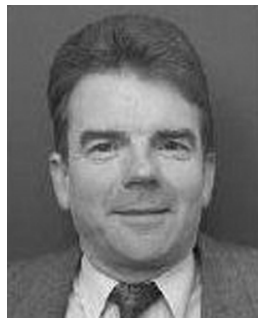

George Stewart received the B.Sc. degree (first class hons.) and the Ph.D. degree for research on integrated optics, both from the University of Glasgow, Glasgow, U.K., in 1974 and 1979, respectively.

$\mathrm{He}$ is currently Professor at the University of Strathclyde, Glasgow, U.K. He continued in integrated optics research at the University of Glasgow until 1985 and then joined the Photonics Group at Strathclyde University to work on fiber optic components, sensors and fiber lasers. Major research areas include the study and design of ion-exchanged optical waveguides, optical fiber surface plasmon polarisers, evanescent field sensors, fiber optic sensors based on laser spectroscopy and fiber lasers for intra-cavity sensors. He is the primary author of 5 chapters in various specialist textbooks and is author or coauthor of more than 120 technical \& scientific papers in the field of fiber and integrated optics. 